\title{
Lefty1 alleviates renal tubulointerstitial injury in mice with unilateral ureteral obstruction
}

\author{
CHANGGENG XU ${ }^{1}$, MINGWEI XU ${ }^{2}$, WEI WANG ${ }^{3}$ and JIE ZHANG ${ }^{3,4}$ \\ ${ }^{1}$ Department of Urology, Wuhan Central Hospital, Wuhan, Hubei 430014; ${ }^{2}$ Department of Urology, \\ Xiangyang Central Hospital, Xiangyang, Hubei 441021; ${ }^{3}$ Department of Urology, Renmin Hospital of Wuhan University, \\ Wuhan, Hubei 430060; ${ }^{4}$ Department of Urology, Huangshi Central Hospital, Hubei Polytechnic University, \\ Huangshi, Hubei 435000, P.R. China
}

Received December 7, 2014; Accepted August 27, 2015

DOI: $10.3892 / \mathrm{mmr} .2015 .4631$

\begin{abstract}
Lefty is a member of the transforming growth factor (TGF) $\beta$ superfamily, which is implicated in left-right patterning during embryogenesis. Previous studies revealed that lefty attenuates the epithelial-mesenchymal transition in tubular epithelial cells. In the present study, the protective effect of leftyl on renal interstitial injury was further assessed. Mice with a unilateral ureteral obstruction (UUO) were sacrificed on days 3,5 and 7 following surgery, and the association between the expression of leftyl and the degree of interstitial fibrosis was investigated. Subsequently, mice with a UUO were administered recombinant leftyl $(300 \mu \mathrm{g} / \mathrm{kg}$ body weight) or vehicle $(0.9 \%$ saline solution; $100 \mu \mathrm{l})$ through tail-vein injection every other day for 6 days. The effects of lefty1 were assessed by measuring the degree of tubulointerstitial fibrosis, tubular injury and atrophy, and also by monitoring the expression levels of $\alpha$-smooth muscle actin ( $\alpha$-SMA), TGF- $\beta 1$, phosphorylated (p)-Smad2/3, kidney injury molecular-1 and endogenous leftyl. The expression of leftyl in the kidney decreased in a time-dependent manner in mice with a UUO, which was inversely correlated with the degree of renal interstitial fibrosis. Furthermore, compared with vehicle treatment, leftyl attenuated renal interstitial fibrosis. Ureteral ligation induced increased expression levels of $\alpha$-SMA, TGF- $\beta 1$ and $p-S m a d 2 / 3$. However, these effects were reduced following treatment with leftyl. The UUO also induced tubular injury and atrophy, whereas leftyl treatment exerted a marked suppressive effect on tubular injury. In addition, exogenous leftyl administered to mice restored the endogenous expression levels of lefty1. The present study demonstrated that leftyl attenuated renal interstitial injury by inhibiting the Smad-dependent TGF- $\beta 1$ signaling pathway.
\end{abstract}

Correspondence to: Professor Jie Zhang, Department of Urology, Renmin Hospital of Wuhan University, 99 Zhangzhidong Road, Wuhan, Hubei 430060, P.R. China

E-mail: zhangjieurology@126.com

Key words: renal interstitial fibrosis, lefty1, TGF- $\beta 1$
Leftyl may therefore by a putative therapeutic agent in the treatment of renal injury.

\section{Introduction}

Chronic renal diseases (CKDs), irrespective of the means of the initial insult, relentlessly progress to end-stage kidney disease, with an irreversible loss of renal architecture and function (1). Renal tubulointerstitial injury is considered to be the hallmark of progressive kidney disease, and the extent of the injury is inversely correlated with renal function. Renal tubulointerstitial injury is histopathologically characterized by an accumulation of myofibroblasts, the marked deposition of extracellular matrix and tubular atrophy (2). Although the molecular mechanisms underlying interstitial injury have been partly elucidated (3), only a few therapies are currently used clinically. Therefore, specific treatments to target interstitial injury are urgently required.

Among the signaling pathways associated with renal tubulointerstitial injury, the Smad-mediated transforming growth factor (TGF)- $\beta 1$ signaling pathway occupies a central role in this process (4). In experimental models and human kidney diseases, TGF- $\beta 1$ is markedly upregulated, and $\mathrm{Smad} 2 / 3$ is highly activated in the fibrotic kidney (5).

The underlying mechanisms by which TGF- $\beta 1$ mediate renal injury are as follows: i) TGF- $\beta 1$ markedly induces the proliferation and activation of renal fibroblasts during renal fibrosis $(5,6)$; ii) TGF- $\beta 1$ elicits the production of the extracellular matrix (ECM) through Smad-dependent mechanisms (7); iii) TGF- $\beta 1$ inhibits ECM degradation by suppressing matrix metalloproteinases and inducing the tissue inhibitor of metalloproteinase (8); and iv) TGF- $\beta 1$ is involved in tubular deletion $(9,10)$.

Lefty is a member of the TGF- $\beta$ superfamily with two variants, which are designated as leftyA and leftyB in humans (11), and lefty1 and lefty 2 in mice (12). Initially, a study by Meno et al (13) revealed that lefty was involved in the formation of embryonic lateral patterning. Ulloa et al (14) demonstrated that leftyA inhibited the phosphorylation of Smad2/3, inhibited the heterodimerization of R-Smads with Smad4, and also inhibited the nuclear translocation of the Smad complex (14). In addition, it was demonstrated that 
leftyA markedly reduced the quantity of collagen deposition, and promoted collagenolysis (15). It was also demonstrated that leftyA or leftyl attenuated the epithelial-mesenchymal transition (EMT) by inhibiting TGF- $\beta 1$ signaling in the mouse HK2 tubular epithelial (16), or the rat NRK52E tubular epithelial (17) cell lines, respectively. Due to the active involvement of the TGF- $\beta 1$ signaling pathway in renal injury, it was hypothesized that leftyl may alleviate renal interstitial injury.

Using a mouse model of unilateral ureteral obstruction (UUO), a well-established experimental model of renal injury characterized by progressive tubulointerstitial fibrosis and tubular atrophy, the present study aimed to assess the ability of leftyl to protect against renal injury, and to determine the possible underlying mechanisms.

\section{Materials and methods}

Experimental design. Male C57BL/6 mice (weighing, 20 g) were obtained from the Experimental Animal Center of Wuhan University (Wuhan, China). The mice were granted free access to water and chow, and were kept under a 12-h light/dark cycle, at $24^{\circ} \mathrm{C}$ and $50 \%$ humidity. The UUO was performed using a previously described method (18). Briefly, under sodium pentobarbital anesthesia $(60 \mathrm{mg} / \mathrm{kg}$ body weight; Merck, Darmstadt, Germany), a complete ureteral obstruction was performed by ligating the left ureter with 4-0 silk (Tianhou Medical Co., Jinan, China). Sham-operated mice had their ureters exposed and manipulated, although they were not ligated. To study the correlation between the expression of leftyl and the degree of interstitial fibrosis, 20 mice were randomly assigned to four groups: i) Sham-operation; ii) UUO for 3 days; iii) UUO for 5 days; iv) UO for 7 days ( $\mathrm{n}=5 /$ group). The mice were subsequently sacrificed on days 3 , 5 and 7 under anesthesia and were fixed by perfusion through the left ventricle. To assess the effects of leftyl on renal tubulointerstitial injury, 18 mice were divided into three groups: i) Sham-operation; ii) UUO plus vehicle treatment; iii) UUO plus leftyl treatment. On the day following surgery, the mice were administered recombinant mouse leftyl (R\&D Systems, Inc., Minneapolis, MN, USA) through a tail-vein injection at a dose of $300 \mu \mathrm{g} / \mathrm{kg}$ body weight, and subsequently on every other day for up to 6 days. The control mice received an injection of an identical volume of vehicle $(0.9 \%$ saline solution; $100 \mu \mathrm{l}$ ). On day 7 following surgery, the mice were sacrificed, and the kidneys were removed. One half of each of the kidneys was fixed in $4 \%$ buffered paraformaldehyde for $24 \mathrm{~h}$ for histological studies; the other half was snap-frozen in liquid nitrogen, and stored at $-80^{\circ} \mathrm{C}$ prior to protein or mRNA extraction. All experiments involving the mice were performed according to institutional guidelines and the protocol of the present study was approved by The Animal Research Ethics Committee of Wuhan University (Wuhan, China).

Histological studies. Kidney sections from the paraffin-embedded tissues were prepared at a thickness of $5 \mu \mathrm{m}$. The sections were stained with periodic acid - Schiff reagent (Biyuntian, Wuhan, China) to assess the extent of tubular atrophy. Picrosirius red (PSR; Biyuntian) staining was used to identify the interstitial collagen. Rabbit polyclonal anti-human lefty1 (cat. no. ab22569; 1:200), rabbit polyclonal anti-human collagen I (cat. no. ab34710; 1:100), rabbit polyclonal anti-human fibronectin (cat. no. ab2413; $1: 400$ ) and rabbit polyclonal anti-human anti-kidney injury molecular-1 (Kim-1; cat. no. ab47635; 1:100) antibodies were purchased from Abcam (Cambridge, UK). Rabbit polyclonal anti-human $\alpha$-smooth muscle actin (SMA) antibody (cat. no. BM0002; 1:50) was purchased from Boshide Bioengineering Co., Ltd. (Wuhan, China). The primary antibodies were incubated for $1 \mathrm{~h}$ at room temperature, or at $4^{\circ} \mathrm{C}$ overnight. Goat polyclonal anti-rabbit IgG (cat. no. BA1055; 1:200; Boshide Bioengineering Co., Ltd.) was used as the secondary antibody. The antigen-specific, positive cells were visualized using streptavidin peroxidase reagents purchased from Boshide Bioengineering Co., Ltd. Brown staining taken up by the cells was indicative of a positive result.

Semi-quantitative analysis for the histological studies. A total of 20 random, non-overlapping fields (magnification, x400) from each animal was selected for assessing PSR staining. These were quantified by assessing the size of the positive area as a percentage of the total area. A total of 10 random, non-overlapping fields (magnification, x400) of each animal were assessed for evaluating tubular Kim-1 staining. These were quantified by counting the positive tubules as a percentage of the total. A total of 10 random, non-overlapping fields (magnification, x200) of each animal were selected for evaluating the tubular atrophy. Tubular atrophy was scored on a scale between 0 and 3, as previously reported (19), although with certain modifications: 0 , absent (no atrophy); 1 , mild (percentage of atrophied tubules, 1-15\%); 2, moderate (percentage of atrophied tubules, 16-30\%); 3, severe (percentage of atrophied tubules, $>30 \%$ ).

Western blot analysis. The kidney tissues were homogenized in a lysis buffer containing $20 \mathrm{mM}$ Tris ( $\mathrm{pH} 7.5$ ), $150 \mathrm{mM} \mathrm{NaCl}$, $1 \%$ Triton $\mathrm{X} 100$, sodium pyrophosphate, $\beta$-glycerophosphate, EDTA, $\mathrm{Na}_{3} \mathrm{VO}_{4}$ and leupeptin (Biyuntian, Wuhan, China) on ice. The lysates were centrifuged at $12,000 \mathrm{x} \mathrm{g}$ at $4^{\circ} \mathrm{C}$ for $20 \mathrm{~min}$ and the protein concentration was determined using a bicinchoninic acid (BCA) protein assay kit. A total of $40 \mu \mathrm{g}$ protein was separated by 12 or $8 \%$ SDS-PAGE (Boshide, Wuhan, China). The proteins were electrotransferred onto a nitrocellulose membrane (Merck-Millipore, Billerica, MA, USA). Non-specific binding to the membrane was blocked for $1 \mathrm{~h}$ at room temperature using $5 \%$ non-fat milk or bovine serum albumin (Boshide). The membranes were subsequently incubated overnight at $4^{\circ} \mathrm{C}$ with the primary antibodies in blocking buffer. Antibodies against TGF- $\beta 1$ (cat. no. sc-146, 1:500), phosphorylated (p-)Smad2/3 (cat. no. sc-11769; 1:400) and $\beta$-actin (cat. no. sc-1619; 1:200) were purchased from Santa Cruz Biotechnology, Inc. (Dallas, TX, USA). Anti-collagen I (cat. no. bs7158R; 1:100) antibody was purchased from Beijing Boao Sen Biotechnology Co., Ltd. (Beijing, China). The sources of the other antibodies used in the western blot analysis [anti-lefty1 (1:400), anti-fibronectin (1:400) and anti- $\alpha$-SMA (1:200) antibodies] were as described above. All antibodies were rabbit polyclonal anti-human antibodies. Subsequently, blots were incubated with the following secondary antibodies: Horseradish peroxidase-conjugated goat anti-rabbit immunoglobulin (Ig)G (cat no. sc-2004; 1:5,000 dilution; Santa 

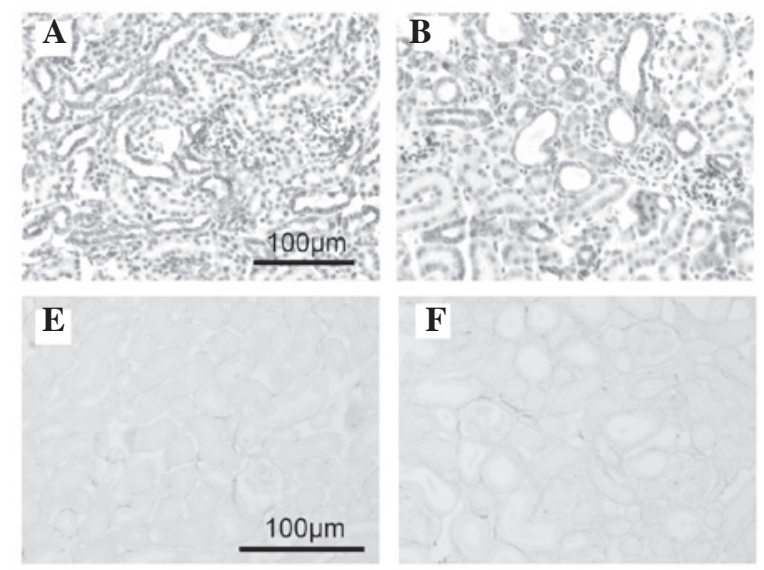

$\mathbf{F}$

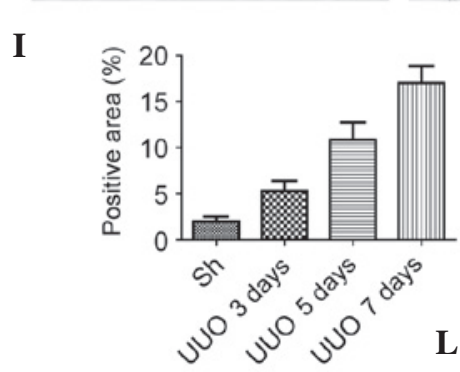

\section{I}

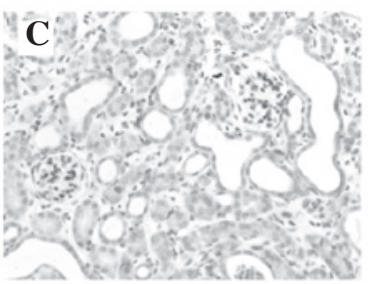

G
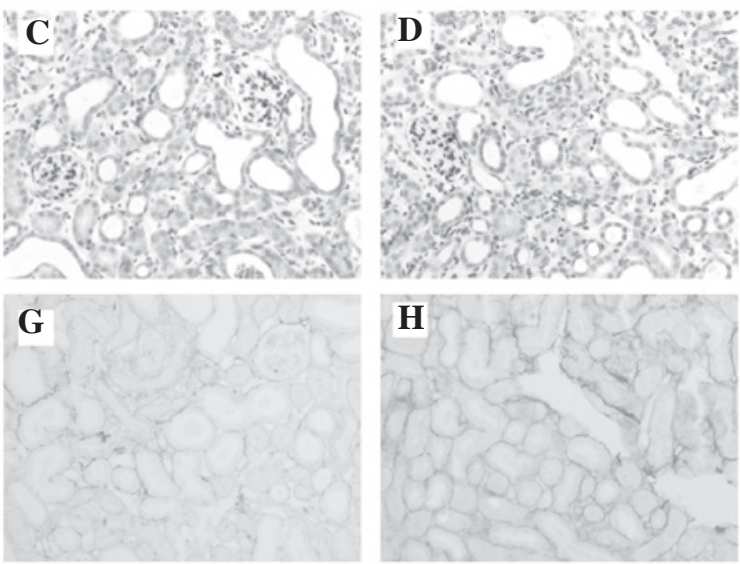

$\mathbf{J}$

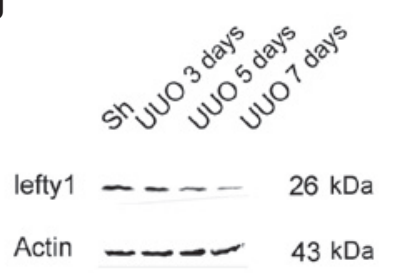

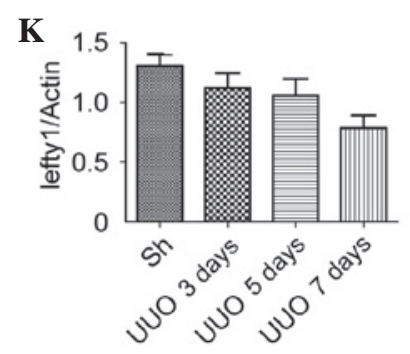

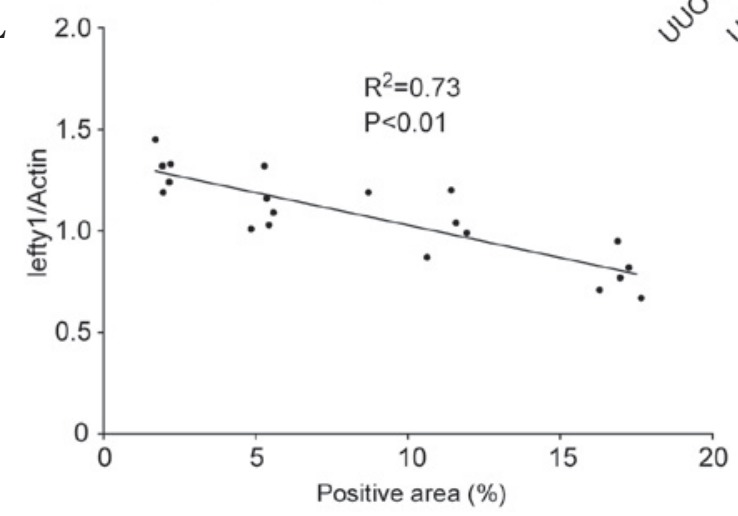

Figure 1. Protein expression of lefty1 in the kidneys of mice with a UUO. (A-D) Representative immunohistochemical staining and (E-H) representative PSR staining of leftyl is shown. (A and E) Sh; (B and F) UUO 3 days; (C and G) UUO 5 days; (D and H) UUO 7 days (Scale bar, $100 \mu \mathrm{m}$ ). (I) Semi-quantitative analysis of the PSR staining was performed. (J) A representative western blot and (K) semi-quantitative analysis for the protein expression of leftyl. (L) The correlation between the expression level of leftyl and the percentage extent of renal fibrosis is illustrated in a scatter plot. All data are expressed as the mean \pm standard deviation ( $\mathrm{n}=5$ /group). PSR, picrosirius red; Sh: Sham-operation group; UUO unilateral ureteral obstruction.

Cruz Biotechnology, Inc.) or IRDye ${ }^{\circledR}$ infrared dye-conjugated anti-rabbit IgG (cat no. 611-1202; 1:5,000 dilution (Rockland Immunochemicals, Inc., Gilbertsville, PA, USA). The bands were visualized using an enhanced chemiluminescence detection kit (GE Healthcare, Little Chalfont, UK) and the Odyssey ${ }^{\circledR}$ imaging system (Li-Cor Biosciences, Lincoln, NE, USA).

Reverse transcription-quantitative polymerase chain reaction $(R T-q P C R)$. RT-qPCR was performed to assess the transcription levels of lefty1. The total RNA was extracted using TRIzol (Invitrogen, Thermo Fisher Scientific, Waltham, MA, USA) and $1 \mu \mathrm{g}$ aliquots of RNA were used in the RT reaction with M-MuLV reverse transcriptase (cat. no. K1621; Thermo Fisher Scientific). The resulting cDNA was used as a template for qPCR analysis. The primers were obtained from Sangon Biological Engineering Technology and Services, Co., Ltd. (Shanghai, China), and the specific primers were designed as follows: Lefty1, sense: 5'-ACTCAAGACCCTTTC AGGACAC-3' and antisense: 5'-CAGCAGAGCCACAGG
AATG-3'; for glyceraldehyde-3-phosphate dehydrogenase, sense: 5'-GGTGAAGGTCGGTGTGAACG-3' and antisense: 5'-CTCGCTCCTGGAAGATGGTG-3'. A $20 \mu 1$ sample of PCR reaction solution, which included $\mathrm{SYBR}^{\circledR}$ Green PCR Master mix (cat. no. RR420A; Takara Bio, Inc., Dalian, China), was amplified according to the manufacturer's instructions with the following thermocycling conditions: Initial denaturation at $94^{\circ} \mathrm{C}$ for $5 \mathrm{~min}$, followed by 40 cycles of denaturation at $94^{\circ} \mathrm{C}$ for $20 \mathrm{sec}$, primer annealing at $58^{\circ} \mathrm{C}$ for $30 \mathrm{sec}$ and elongation at $72^{\circ} \mathrm{C}$ for $45 \mathrm{sec}$. The reactions were performed on an ABI PRISM ${ }^{\circledR} 7500$ Sequence Detection system (Applied Biosystems Life Technologies, Beijing, China). The calculations of the relative changes in the mRNA expression levels were performed using the $2^{-\Delta \Delta \mathrm{Ct}}$ method (20).

Statistical analysis. The data are expressed as the mean \pm standard deviation. One-way analysis of variance, followed by the Student-Newman-Keuls test, was used for the quantitative data, whereas the Kruskal-Wallis test was used for 

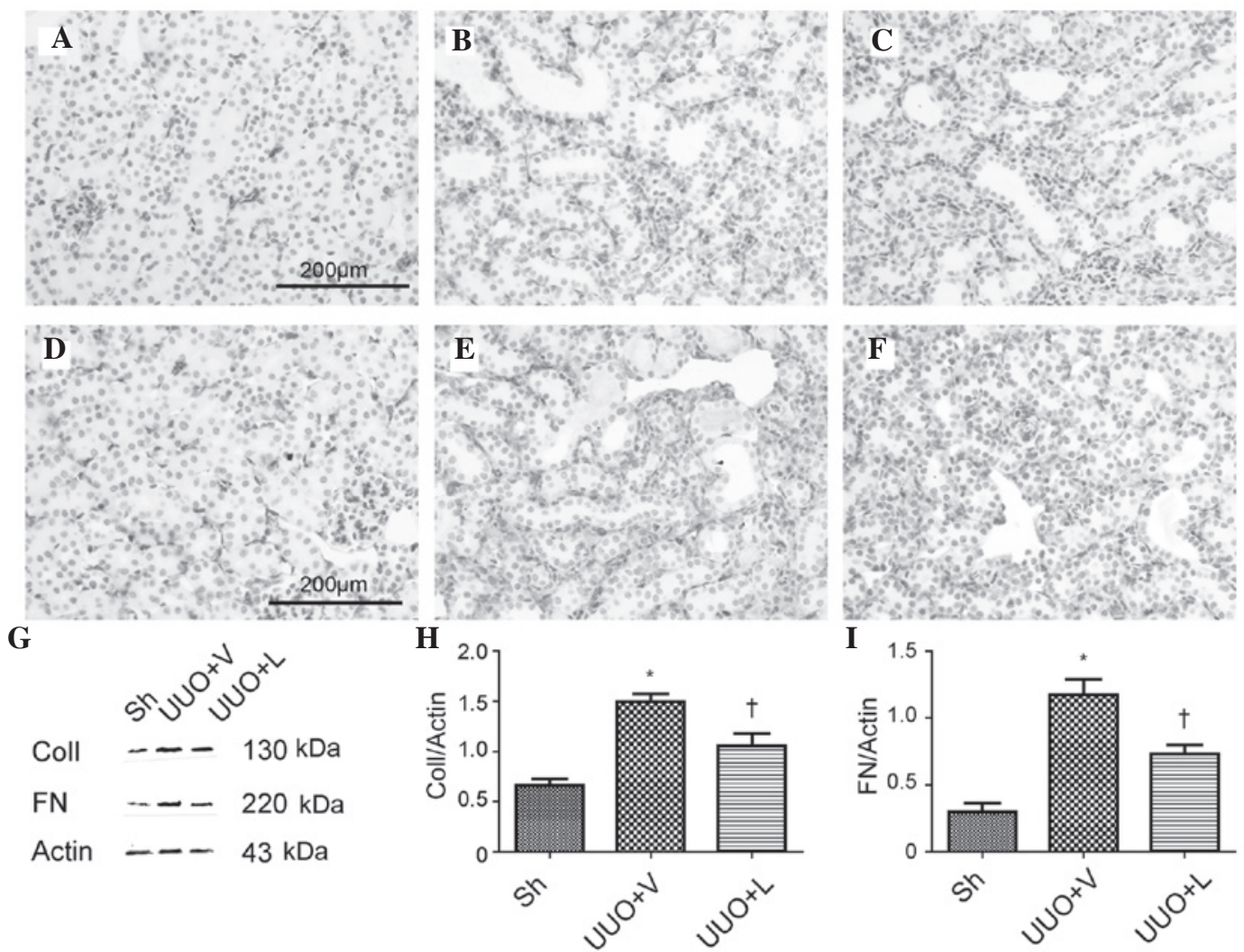

Figure 2. Effects of administering exogenous leftyl on the UUO-induced renal fibrosis. Representative micrographs of (A-C) Col I and (D-F) FN staining in the kidney tissue of mice are shown. (A and D) SH mice; (B and E) UUO + V; (C and F) UUO + L (Scale bar, $200 \mu \mathrm{m}$ ). (G) A representative western blot and the resultant semi-quantitative analysis of (H) Coll and (I) FN are shown. All data are expressed as the mean \pm standard deviation ( $\mathrm{n}=6 / \mathrm{group} ;{ }^{*} \mathrm{P}<0.05$, vs. Sh; ${ }^{\dagger} \mathrm{P}<0.05$, vs. UUO + V). Sh, sham-operation; UUO, unilateral ureteral obstruction; V, vehicle; L, lefty1; Coll, collagen I; FN, fibronectin.

non-normally distributed data. Statistical analyses of the data were performed using GraphPad Prism (version 5.0; GraphPad Software, Inc., La Jolla, CA, USA). P $<0.05$ was considered to indicate a statistically significant difference.

\section{Results}

Expression ofleftyl is inversely correlated with renalfibrosis. The protein expression of leftyl in the kidneys of mice with a UUO were investigated. As shown in Fig. 1A-D, the immunohistochemical (IHC) staining demonstrated that leftyl was predominantly localized in the tubular epithelial cells and that the expression levels of lefty1 were gradually decreased in the obstructed kidneys in a time-dependent manner (up to 7 days following the UUO).. Furthermore, during the first week of the UUO, the degree of the renal interstitial fibrosis increased, as assessed by the PSR staining experiments (Fig. 1E-I). Western blotting was used to corroborate these results (Fig. 1J). A semi-quantitative analysis of the western blotting results revealed that the protein expression of leftyl in the kidneys of the mice in the sham-operation and UUO for 3, 5 or 7 day groups (normalized against $\beta$-actin) were $1.31 \pm 0.10,1.12 \pm 0.13,1.06 \pm 0.14$ and $0.78 \pm 0.11$, respectively (Fig. 1K). Subsequently, the correlation between the protein expression of leftyl and the degree of renal fibrosis was examined. The percentage of positive area in the kidneys of mice in the sham-operation and UUO for 3, 5 or 7 day groups were $1.99 \pm 0.51,5.30 \pm .08,10.85 \pm 1.90$ and $17.01 \pm 1.85 \%$, respectively (Fig. 1L). A linear regression plot revealed a close correlation $\left(R^{2}=0.73 ; \mathrm{P}<0.01\right)$ between the protein expression of lefty1 and the degree of renal fibrosis (Fig. 1K). These results indicated that the protein expression of lefty1 was inversely correlated with the degree of renal interstitial fibrosis.

Leftyl attenuates the degree of interstitial fibrosis. Due to the inverse correlation between the protein expression of leftyl and the extent of interstitial fibrosis, exogenous recombinant leftyl was administered to mice with a UUO to examine whether leftyl attenuates interstitial fibrosis. The IHC staining patterns for collagen I and fibronectin are shown in Fig. 2A-C and Fig. 2D-F, respectively. In comparison with the sham-operated group, the protein expression levels of collagen I and fibronectin were significantly increased in mice on treatment with vehicle, which decreased following treatment with leftyl. These findings were corroborated by western blot analysis. Compared with the sham-operated group, the protein expression levels of collagen I were significantly increased $(1.49 \pm 0.09$, vs. $0.67 \pm 0.07 ; \mathrm{P}<0.05)$, which were subsequently diminished upon treatment with leftyl $(1.06 \pm 0.11$, vs. $1.49 \pm 0.09 ; \mathrm{P}<0.05)$. Furthermore, the UUO led to an increased protein expression of fibronectin $(1.17 \pm 0.12$, vs. $0.30 \pm 0.07 ; \mathrm{P}<0.05)$, which was reduced following administration of leftyl $(0.73 \pm 0.07$, vs. $1.17 \pm 0.12$; $\mathrm{P}<0.05)$. These results indicated that leftyl attenuated renal interstitial fibrosis in mice with a UUO. 

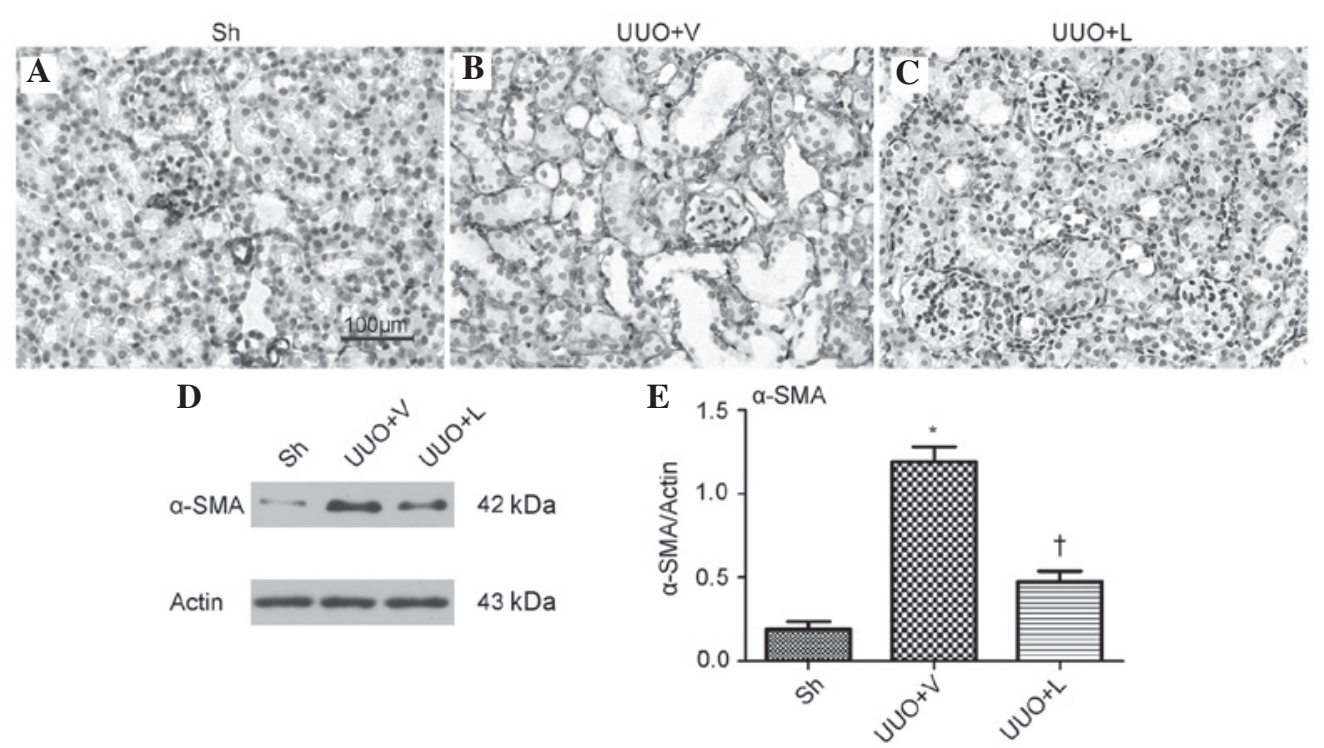

Figure 3. Lefty1 reduces the levels of renal $\alpha$-SMA in the obstructed kidney following a UUO. Representative micrographs of (A-C) immunohistochemical staining (Scale bar, $100 \mu \mathrm{m}$ ) and (D) western blotting are shown. (E) Semi-quantitative analysis was performed for the western blotting and $\beta$-actin was used as the internal loading control. All data are expressed as the mean \pm standard deviation ( $\mathrm{n}=6 /$ group; ${ }^{*} \mathrm{P}<0.05$, vs. Sh; ${ }^{\mathrm{P}} \mathrm{P}<0.05$, vs. UUO + V. Sh, sham-operation; UUO, unilateral ureteral obstruction; V, vehicle; L, leftyl. $\alpha$-SMA, $\alpha$-smooth muscle actin.

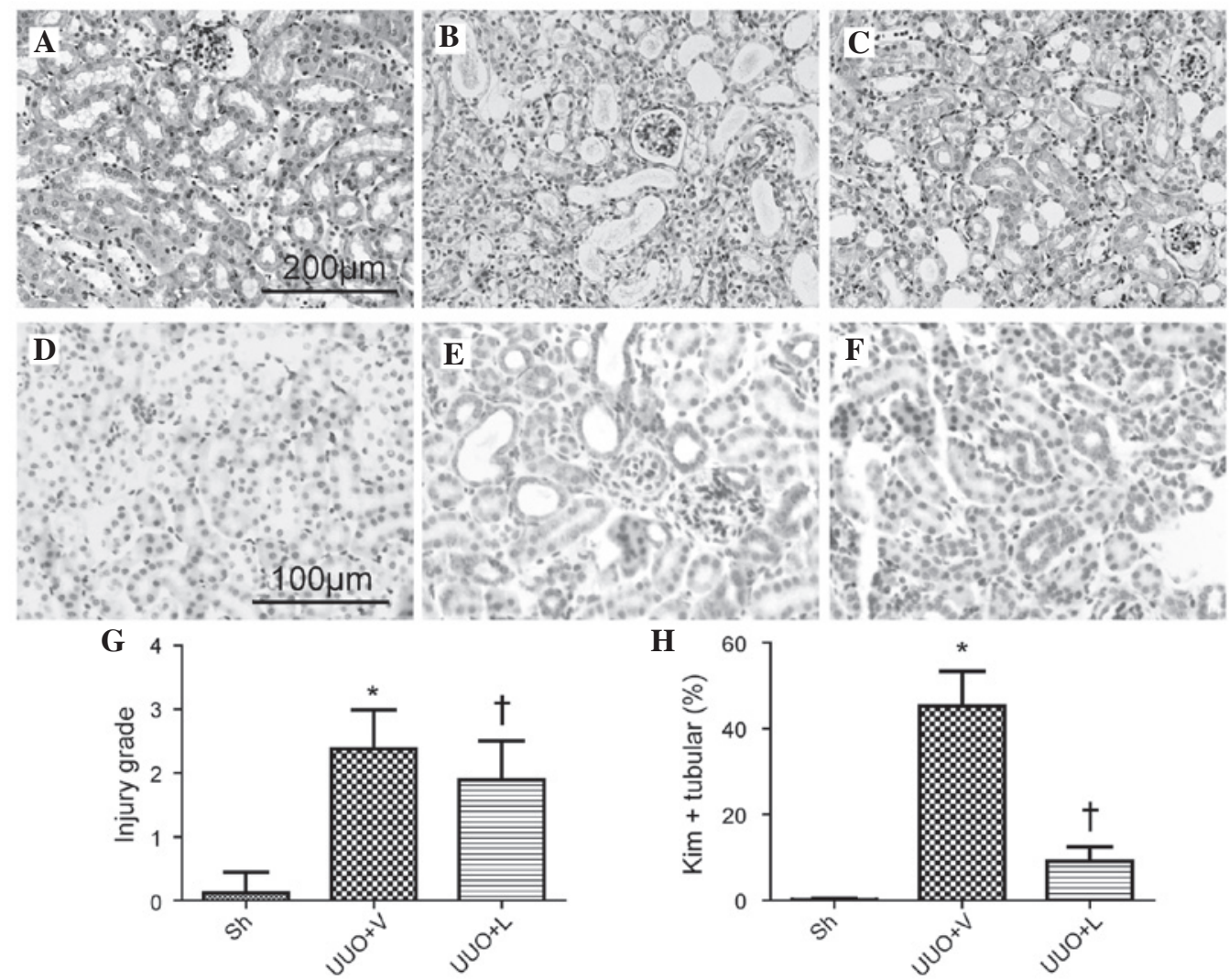

Figure 4. Lefty1 attenuates tubular atrophy and injury. (A-C) Representative micrographs of the periodic acid shiff staining experiments are shown (Scale bar, $200 \mu \mathrm{m}$ ); (D-F) show the representative micrographs of the Kim-1 staining experiments (Scale bar, $100 \mu \mathrm{m}$ ). The semi-quantitative histograms for (G) the injury grade and $(\mathrm{H})$ the percentage of Kim-1-positive cells are shown. All data are expressed as the mean \pm standard deviation ( $\mathrm{n}=6 / \mathrm{group}$; ${ }^{*} \mathrm{P}<0.05$, vs. Sh; ${ }^{\top} \mathrm{P}<0.05$, vs. UUO + V. Kim(-1), kidney injury molecular-1; Sh, sham-operation; UUO + V, unilateral ureteral obstruction plus treatment with vehicle; UUO + L, UUO plus treatment with leftyl.

Leftyl suppresses the accumulation of myofibroblasts. $\alpha$-SMA is a marker for myofibroblasts, which are the major class of ECM-producing cells. Therefore it was hypothesized that treatment with lefty1 may suppress the accumulation of myofibroblasts. As shown in Fig. 3A-C, IHC staining demonstrated that $\alpha$-SMA was localized in the vascular smooth 

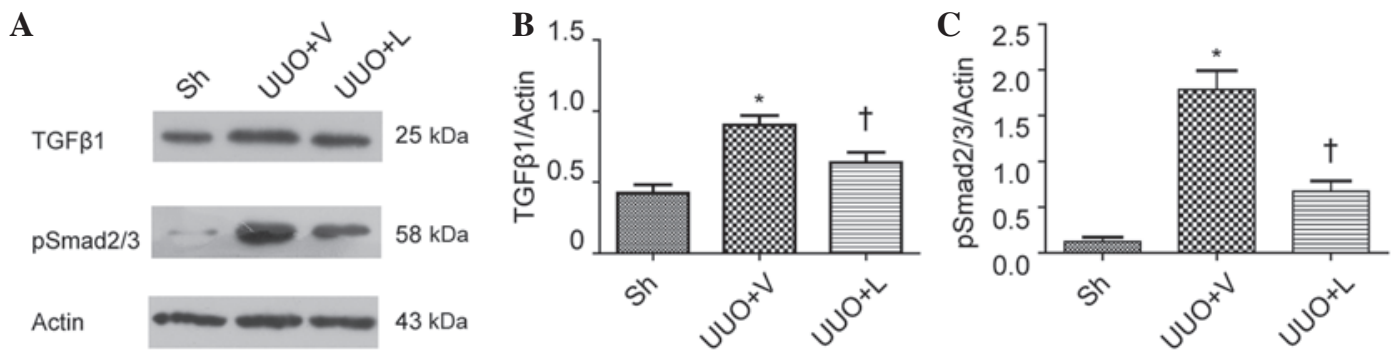

Figure 5. Effects of lefty1 on the protein expression levels of renal TGF- $\beta 1$ and pSmad2/3. (A) A representative western blot analysis is shown $(B$ and $C)$ Semi-quantitative histograms were derived from the western blotting data. $\beta$-actin was used as an internal loading control. All data are expressed as the mean \pm standard deviation ( $\mathrm{n}=6$ /group; ${ }^{*} \mathrm{P}<0.05$, vs. $\mathrm{Sh}$; ${ }^{\mathrm{P}}<0.05$, vs. $\mathrm{UUO}+\mathrm{V}$ ). Sh, sham-operation; UUO, unilateral ureteral obstruction; V, vehicle; $\mathrm{L}$, leftyl; p, phosphorylated; TGF- $\beta$, transforming growth factor- $\beta 1$.

A

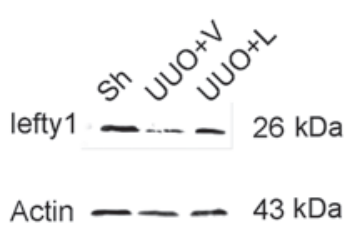

B

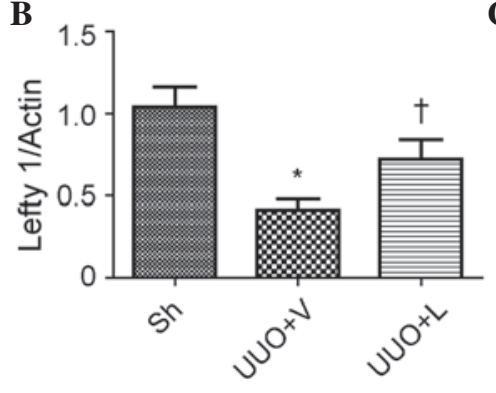

C

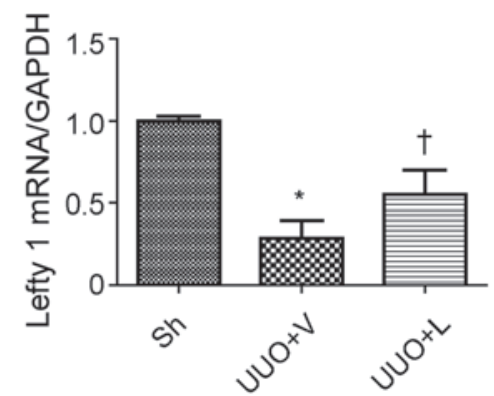

Figure 6. Exogenous leftyl restores the expression of endogenous leftyl. (A) A representative western blot gel, and the resultant semi-quantitative analyses of (B) the protein and (C) the mRNA expression levels for leftyl are shown. Either $\beta$-actin or GAPDH were used as the internal control. All data are expressed as the mean \pm standard deviation ( $\mathrm{n}=6$ /group; ${ }^{*} \mathrm{P}<0.05$, vs. Sh; ${ }^{\dagger} \mathrm{P}<0.05$, vs. UUO+V). Sh, sham-operation; UUO, unilateral ureteral obstruction; V, vehicle; L, lefty1. GAPDH, glyceraldehyde-3-phosphate dehydrogenase.

muscle cells in the normal mouse kidney. Compared with the sham-operated group, a large quantity of $\alpha$-SMA-positive cells were localized in the peritubular interstitial space in the UUO mice, which were treated with vehicle, and the subsequent treatment with leftyl decreased the protein expression of $\alpha$-SMA. Western blot analysis also revealed a markedly increased expression of $\alpha$-SMA in the UUO mice, which were treated with vehicle, compared with the sham-operated mice $(1.19 \pm 0.09$, vs. $0.19 \pm 0.05 ; \mathrm{P}<0.05$; Fig. $3 \mathrm{D}$ and E). Treatment with leftyl resulted in a significant reduction in the protein expression levels of $\alpha$-SMA compared with the UUO plus vehicle treatment group $(0.47 \pm 0.07$, vs. $1.19 \pm 0.09 ; \mathrm{P}<0.05)$. These results indicated that leftyl suppressed the accumulation of myofibroblasts.

Leftyl ameliorates tubular injury and atrophy. Tubular atrophy is a prominent feature in tubulointerstitial injury. In comparison with the sham-operated group, the UUO plus vehicle treatment group exhibited severe tubular atrophy $(2.38 \pm 0.16$, vs . $0.12 \pm 0.32 \% ; \mathrm{P}<0.05)$. However, lefty 1 suppressed tubular atrophy $(1.90 \pm 0.60$, vs. $2.38 \pm 0.16 \%$; $\mathrm{P}<0.05$; Fig. 4A-C) in the obstructed kidney. Since tubular atrophy is a histopathological manifestation of tubular injury, it was hypothesized whether leftyl inhibited tubular damage, and staining with Kim-1, an indicator of tubular damage, was used to assess this. In comparison with the sham-operated group, the UUO plus vehicle treatment group exhibited more Kim-1-positive tubules (45.27 \pm 8.12 , vs. $0.12 \pm 0.32 \%$; $\mathrm{P}<0.05)$. Lefty1 significantly suppressed tubular injury (9.12 \pm 3.31 , vs. $45.27 \pm 8.12 \%$; $\mathrm{P}<0.05$; Fig. 4D-F) in the obstructed kidney. These results indicated that leftyl ameliorated tubular atrophy and injury.

Leftyl inhibits the Smad-dependent TGF- $\beta 1$ signaling pathway. Due to the evidence that the Smad-dependent TGF- $\beta 1$ signaling pathway occupies a central role in renal injury, whether treatment with leftyl inhibited this pathway was subsequently investigated. Western blot analysis revealed that the protein expression levels of TGF- $\beta 1$ in the obstructed kidney was significantly increased compared with the sham group $(0.90 \pm 0.07$, vs. 0.42 \pm 0.06 ; $\mathrm{P}<0.05$; Fig. 5A and $\mathrm{B})$. Treatment with leftyl reduced the protein expression of TGF- $\beta 1$ (0.64 \pm 0.07 , vs. $0.90 \pm 0.07$; $\mathrm{P}<0.05$; Fig. $5 \mathrm{~B})$. In addition, the protein expression of $\mathrm{p}-\mathrm{Smad} 2 / 3$ in the obstructed kidney was considerably increased compared with the sham group $(1.78 \pm 0.21$, vs. $0.12 \pm 0.05 ; \mathrm{P}<0.05)$, and the subsequent treatment with lefty1 also diminished the protein expression of p-Smad2/3 (0.67 \pm 0.11 , vs. $1.78 \pm 0.21 ; \mathrm{P}<0.05$; Fig. 5C). These results indicated that leftyl inhibited the Smad-dependent TGF- $\beta 1$ signaling pathway.

Exogenously administered leftyl restores the expression of endogenous leftyl. Finally, whether exogenously administered lefty1 restored the endogenous expression of leftyl was investigated (Fig. 5). Compared with the sham group, the UUO plus vehicle treatment group demonstrated a reduced protein expression of lefty1 $(0.42 \pm 0.07$, vs. $1.04 \pm 0.12$; $\mathrm{P}<0.05$; Fig. 5B). However, leftyl treatment augmented the expression of lefty1 $(0.42 \pm 0.07$, vs. $0.72 \pm 0.12 ; \mathrm{P}<0.05)$. At the mRNA expression level, the UUO plus vehicle treatment group exhibited a 
decreased mRNA expression of leftyl $(0.29 \pm 0.11$, vs. $1.00 \pm 0.03$; $\mathrm{P}<0.05$; Fig. 5C). Nevertheless, lefty1 treatment restored lefty1 expression $(0.55 \pm 0.15$, vs. $0.29 \pm 0.11 ; \mathrm{P}<0.05)$.

\section{Discussion}

Due to the high level of homology between human leftyA and $\mathrm{B}$, and the mouse leftyl and 2 proteins (11), it is conceivable that they may share a similar function. An in vitro study, reported previously by our laboratory, revealed that leftyA attenuated the EMT in a tubular cell line by dampening TGF- $\beta 1$-mediated signal transduction (16). These results were confirmed by another previous study (17). Despite its antagonism towards TGF- $\beta 1$ signaling in vitro, whether or not lefty exerted an identical effect in vivo remained to be elucidated. The present study demonstrated for the first time, to the best of our knowledge, that leftyl is a protective regulator during the UUO process. In UUO-challenged mice, the lefty1 protein was specifically downregulated by ureteral ligation, and treatment with leftyl restored the expression of endogenous leftyl in the obstructed kidney. Furthermore, treatment with leftyl also decreased the extent of tubular injury and interstitial fibrosis. At the mechanistic level, the present study revealed that TGF- $\beta 1$-mediated signal transduction was mitigated by lefty1.

Lefty was initially characterized as a diffusible morphogen, which is transiently expressed in the left half of gastrulating mouse embryos (13). In adult mice, lefty is expressed in the mouse endometrium during the estrous cycle and the peri-implantation period (21). In the present study, the presence of leftyl in the kidney was demonstrated, although the biological function of leftyl in kidney diseases remains to be elucidated. The present study demonstrated that lefty1 is specifically downregulated upon induction by a UUO. Indeed, lefty1 may be regulated by other types of signaling molecules under a variety of conditions, including $\operatorname{Smad} 2 / 3$, FoxH1, forkhead activin signal transducer 2 , Oct $3 / 4$ and microRNAs (22). UUO manipulation generated a diversity of signaling molecules, among which TGF- $\beta 1$ may be ranked as the most important. The in vitro study published previously by our laboratory demonstrated that TGF- $\beta 1$ inhibited lefty expression in a dose-dependent manner (16). Therefore, the UUO-induced downregulation of lefty1 may be partially caused by an overproduction of this cytokine. Notably, in the present study, it was observed that the exogenous administration of leftyl restored the expression of endogenous leftyl at the transcriptional level. The mechanism by which exogenous leftyl regulates the endogenous expression of leftyl remains to be elucidated. Besser (23) demonstrated that the expression of lefty in undifferentiated human embryonic stem cells required the activation of Smad2/3. However, the present study revealed that $\mathrm{p}-\mathrm{Smad} 2 / 3$ was downregulated. This discrepancy may be explained by the different cellular microenvironment, therefore, an investigation into the mechanism underlying the regulation of leftyl following the induction of UUO is required in future studies.

Leftyl may attenuate tubulointerstitial injury, as evidenced by the decreased deposition of the ECM, suppression of the accumulation of the myofibroblasts and the amelioration of tubular atrophy. The identification of leftyl as a key protein in the amelioration of interstitial injury permits an exploration of the underlying mechanism. It is widely accepted that TGF- $\beta 1$ is a key modulator of renal interstitial injury in experimental model and human kidney diseases (9). For example, TGF- $\beta 1$ is markedly upregulated in the fibrotic kidney, regardless of the initial cause of the kidney disease. Furthermore, the overexpression of TGF- $\beta 1$ by renal tubular epithelial cells resulted in tubulointerstitial fibrosis and tubular deletion in the absence of any injury, revealing the functional importance of TGF- $\beta 1$ in CKDs (6). TGF- $\beta 1$ activates several intracellular Smad signaling cascades to fulfil its functions. The present study demonstrated that the upregulation of TGF- $\beta 1$ was induced in the obstructed kidney, whereas treatment with leftyl decreased the expression of TGF- $\beta 1$. Furthermore, lefty1 also diminished the phosphorylation of Smad2/3. Indeed, the injury-promoting effects of TGF- $\beta 1$ were further confirmed by a report which demonstrated that the complete inhibition of TGF- $\beta 1$ by a neutralizing TGF- $\beta 1$ antibody markedly ameliorated renal damage in vivo (24). Furthermore, a deficiency of Smad3 attenuated renal tubular injury following a UUO (10). These results demonstrated that the inhibitory effect of leftyl on the Smad-dependent TGF- $\beta 1$ signaling pathway was responsible for its ability to protect against injury.

It is noteworthy that, in view of the various different functions of lefty, its ability to inhibit renal injury in the obstructed kidneys may be mediated by other mechanisms. Previous studies demonstrated that lefty contributes to the remodeling of the ECM by increasing the collagenolytic, gelatinolytic, elastolytic and caseinolytic activities in vivo (15). In human endometrium, lefty also regulates the expression and activation of matrix metalloproteinase 9 (25). Therefore, increased activities of the matrix degradation enzymes mediated by lefty may provide additional pathways that lead to the suppression of renal fibrogenesis in vivo. In addition, it remains to be determined whether the protective effect of leftyl is associated with the non-Smad-dependent TGF- $\beta 1$ pathway, which is also responsible for renal injury (26). In fact, it is possible that the multiple pathways triggered by leftyl from exogenous sources may work in concert, culminating ultimately in the amelioration of renal injury in the obstructed kidneys in vivo.

It should be noted that TGF- $\beta 1$ is a pleiotropic cytokine involved in a number of cell functions, including cell growth, differentiation and the regulation of immune responses, in addition to the control of the biosynthesis of extracellular connective tissue (27). In the present study, the mice receiving leftyl treatment were active, and there appeared to be no clear side effects. However, in the clinical setting, it remains to be elucidated whether patients exposed to a prolonged treatment with leftyl experience any adverse reactions, including the impairment of immune function or delayed wound healing. Nevertheless, the findings of the present study may have important implications for developing clinically relevant therapeutic strategies for renal injury. The administration of lefty may provide a novel and effective treatment for CKDs by specifically inhibiting the activation of the TGF- $\beta 1$ signaling pathway. Although the protective effect of lefty in vivo remains to be confirmed in other animal model systems and in patients with CKDs, the administration of exogenous lefty protein appears to have the potential to hinder the progression of CKDs, devastating conditions which are incurable at present. 


\section{Acknowledgements}

This study was supported by the National Natural Science Foundation of China (no. 81170710) and the Hospital Foundation for Doctors (no. YB15B01).

\section{References}

1. Meguid El, Nahas A and Bello AK: Chronic kidney disease: The global challenge. Lancet 365: 331-340, 2005.

2. Barnes JL and Glass WF II: Renal interstitial fibrosis: A critical evaluation of the origin of myofibroblasts. Contrib Nephrol 169: 73-93, 2011.

3. Chuang PY, Menon MC and He JC: Molecular targets for treatment of kidney fibrosis. J Mol Med (Berl) 91: 549-559, 2013

4. López-Hernández FJ and López-Novoa JM: Role of TGF- $\beta$ in chronic kidney disease: An integration of tubular, glomerular and vascular effects. Cell Tissue Res 347: 141-154, 2012.

5. Gewin L and Zent R: How does TGF- $\beta$ mediate tubulointerstitial fibrosis? Semin Nephrol 32: 228-235, 2012.

6. Koesters R, Kaissling B, Lehir M, Picard N, Theilig F, Gebhardt R, Glick AB, Hähnel B, Hosser H, Gröne HJ and Kriz W: Tubular overexpression of transforming growth factor-betal induces autophagy and fibrosis but not mesenchymal transition of renal epithelial cells. Am J Pathol 177: 632-643, 2010.

7. Moon JA, Kim HT, Cho IS, Sheen YY and Kim DK: IN-1130, a novel transforming growth factor-beta type I receptor kinase (ALK5) inhibitor, suppresses renal fibrosis in obstructive nephropathy. Kidney Int 70: 1234-1243, 2006.

8. Douthwaite JA, Johnson TS, Haylor JL, Watson P and El Nahas AM: Effects of transforming growth factor-betal on renal extracellular matrix components and their regulating proteins. J Am Soc Nephrol 10: 2109-2119, 1999.

9. García-Sánchez O, López-Hernández FJ and López-Novoa JM: An integrative view on the role of TGF-beta in the progressive tubular deletion associated with chronic kidney disease. Kidney Int 77: 950-955, 2010.

10. Inazaki K, Kanamaru Y, Kojima Y, Sueyoshi N, Okumura K, Kaneko K, Yamashiro Y, Ogawa H and Nakao A: Smad3 deficiency attenuates renal fibrosis, inflammation, and apoptosis after unilateral ureteral obstruction. Kidney Int 66: 597-604, 2004.

11. Kosaki K, Bassi MT, Kosaki R, Lewin M, Belmont J, Schauer G and Casey B: Characterization and mutation analysis of human LEFTY A and LEFTY B, homologues of murine genes implicated in left-right axis development. Am J Hum Genet 64 712-721, 1999.

12. Meno C, Ito Y, Saijoh Y, Matsuda Y, Tashiro K, Kuhara S and Hamada $\mathrm{H}$ : Two closely-related left-right asymmetrically expressed genes, lefty-1 and lefty-2: Their distinct expression domains, chromosomal linkage and direct neuralizing activity in Xenopus embryos. Genes Cells 2: 513-524, 1997.
13. Meno C, Saijoh Y, Fujii H, Ikeda M, Yokoyama T, Yokoyama M, Toyoda $\mathrm{Y}$ and Hamada H: Left-right asymmetric expression of the TGF beta-family member lefty in mouse embryos. Nature 381: 151-155, 1996.

14. Ulloa L and Tabibzadeh S: Lefty inhibits receptor-regulated Smad phosphorylation induced by the activated transforming growth factor-beta receptor. J Biol Chem 276: 21397-21404, 2001.

15. Mason JM, Xu HP, Rao SK, Leask A, Barcia M, Shan J, Stephenson R and Tabibzadeh S: Lefty contributes to the remodeling of extracellular matrix by inhibition of connective tissue growth factor and collagen mRNA expression and increased proteolytic activity in a fibrosarcoma model. J Biol Chem 277: 407-415, 2002.

16. Li Y, Zhang J, Fang L, Luo P, Peng J and Du X: Lefty A attenuates the TGF-betal-induced epithelial to mesenchymal transition of human renal proximal epithelial tubular cells. Mol Cell Biochem 339: 263-270, 2010.

17. Mariasegaram M, Tesch GH, Verhardt S, Hurst L, Lan HY and Nikolic-Paterson DJ: Lefty antagonises TGF-beta1 induced epithelial-mesenchymal transition in tubular epithelial cells. Biochem Biophys Res Commun 393: 855-859, 2010.

18. Sorensen I, Susnik N, Inhester T, Degen JL, Melk A, Haller H and Schmitt R: Fibrinogen, acting as a mitogen for tubulointerstitial fibroblasts, promotes renal fibrosis. Kidney Int 80: 1035-1044, 2011.

19. Li L, Zepeda-Orozco D, Black R and Lin F: Autophagy is a component of epithelial cell fate in obstructive uropathy. Am J Pathol 176: 1767-1778, 2010.

20. Livak KJ and Schmittgen TD: Analysis of relative gene expression data using real-time quantitative PCR and the 2(-Delta Delta C(T)) Method. Methods 25: 402-408, 2001.

21. Tang M, Xu Y, Julian J, Carson D and Tabibzadeh S: Lefty is expressed in mouse endometrium in estrous cycle and peri-implantation period. Hum Reprod 20: 872-880, 2005.

22. Tabibzadeh S and Hemmati-Brivanlou A: Lefty at the crossroads of 'stemness' and differentiative events. Stem Cells 24: 1998-2006, 2006.

23. Besser D: Expression of nodal, lefty-a and lefty-B in undifferentiated human embryonic stem cells requires activation of Smad2/3. J Biol Chem 279: 45076-45084, 2004.

24. Miyajima A, Chen J, Lawrence C, Ledbetter S, Soslow RA, Stern J, Jha S, Pigato J, Lemer ML, Poppas DP, et al: Antibody to transforming growth factor-beta ameliorates tubular apoptosis in unilateral ureteral obstruction. Kidney Int 58: 2301-2313, 2000.

25. Cornet PB, Galant C, Eeckhout Y, Courtoy PJ, Marbaix E and Henriet P: Regulation of matrix metalloproteinase-9/gelatinase $\mathrm{B}$ expression and activation by ovarian steroids and LEFTY-A/endometrial bleeding-associated factor in the human endometrium. J Clin Endocrinol Metab 90: 1001-1011, 2005.

26. Zhang YE: Non-Smad pathways in TGF-beta signaling. Cell Res 19: 128-139, 2009.

27. Lan HY: Diverse roles of TGF- $\beta /$ Smads in renal fibrosis and inflammation. Int J Biol Sci 7: 1056-1067, 2011. 